\title{
Pour l'enseignement d'une grammaire du sens
}

Patrick Charaudeau ${ }^{a}$

\section{Resumo \\ Pelo ensino de uma gramática do sentido}

No artigo, apresenta-se a concepção de uma "gramática do sentido e da expressão" ("enunciativa", "discursiva"e "reflexiva"), como proposta de aplicação da teoria linguística ao ensino de língua (materna ou estrangeira) nas escolas, orientada para o desenvolvimento da proficiência linguística dos alunos. Contrapõe-se a "gramática semântica", orientada para o sentido, à "gramática morfológica", orientada para a descrição das formas linguísticas, a fim de se mostrar a necessidade de se refletir, na escola, acerca da finalidade semântica dos recursos linguísticos e da relação entre as formas e aquilo que significam de acordo com seu emprego. Defende-se a análise das categorias linguísticas como mecanismos de expressão que permitem descrever, narrar e argumentar de acordo com a situação de comunicação.

Palavras-chave: Gramática semântica. Ensino de língua. Recursos linguístico-discursivos. 


\section{Introduction}

Traiter de la question de l'enseignement d'une langue est une affaire délicate. On pourrait rappeler cette banalité que décrire une langue n'est pas la même chose que l'enseigner, mais, outre que notre système d'enseignement semble souvent ignorer cette banalité, il faut pouvoir dire en quoi ce n'est pas la même chose. A cela, il y a au moins deux raisons de poids. La première est qu'il faut accepter la relativité du savoir, et abandonner l'idée qu'il n'existerait qu'un système de description ou d'explication du fonctionnement de la langue. Une explication tire sa validité de son seul cadre théorique, et peut donc être nulle et non avenue au regard d'un autre cadre théorique. Il suffit de lire les actes de tel ou tel colloque sur les notions de "déterminants", de "sujet-thèmeprédication", etc. pour s'en convaincre. Cela est bien normal. Toute activité scientifique est génératrice de points de vue différents ; c'est ainsi qu'avance la recherche ${ }^{1}$, La deuxième raison concerne le problème de l'utilisation d'un savoir dans un champ d'application qui n'est pas celui de son origine. Lobservation montre que telle explication savante peut être soit inutile, soit incompréhensible, soit même déviante dans tel contexte d'application, et l'on dit alors que cette théorie est inapplicable. C'est que tout système explicatif dépend de l'enjeu communicatif dans lequel il s'insère. Que se passe-t-il donc lorsque l'on veut utiliser telle description linguistique dans le champ de l'enseignement de la langue (maternelle ou étrangère). Il se passe que l'enjeu communicatif de l'enseignement n'est pas celui de l'analyse savante: les partenaires de l'échange ne sont pas les mêmes (élèves/professeur dans un cas, chercheurs dans l'autre), la finalité de la communication n'est pas la même (d'enseignement-apprentissage dans un cas, de démonstrationcritique dans l'autre), les circonstances dans lesquelles se produit celle-ci non plus (l'école et la classe avec tout ce que cela suppose sociologiquement dans un cas, les revues et colloques

A ce propos, faisons remarquer qu'il est absurde d'attendre que les linguistes se mettent d'accord pour tirer parti des résultats de leur recherche, ce n'est pas là leur objectif. dans l'autre).

Il faut donc accepter de considérer qu'il y a plusieurs marchés du savoir, des marchés du savoir savant et des marchés du savoir appliqué. Les sciences du langage se trouvent dans le premier avec des enjeux qui lui sont propres ; l'enseignement 
2 Peut-être, est-ce une question de survie, je veux dire une question de représentation sociale, aucune société ne pouvant imaginer une école qui ne serait pas le lieu d'apprentissage d'un savoir savant.

3 Je m'appuie, pour cela, sur mon expérience de dix ans de travail à l'INRP avec des enseignants du primaire et du secondaire.

4 Souvenons-nous des effets de l'application de la grammaire générative dans les années 70 . des langues dans le second qui n'est ni inférieur ni moins noble. Il n'empêche que longtemps a été entretenue - et encore maintenant- une confusion entre le savoir savant sur la langue et le savoir qui doit prévaloir dans le cadre de l'enseignement. Cela ne date pas d'aujourd'hui. Dès le début de l'apparition des grammaires, est née l'idée qu'une grammaire représentait l'identité (voire le génie) d'un peuple, et que, conséquemment, c'est celle-ci qui devait être enseignée à l'école ${ }^{2}$. Pourtant, la description que toute grammaire propose de sa langue n'a rien d'absolu, car elle dépend des objectifs que l'on se donne et des outils que l'on utilise. Il n'y a donc pas une, mais plusieurs grammaires d'une langue. En raison des deux marchés du savoir, il y a toujours eu deux types d'ouvrages grammaticaux: les uns dits scolaires, les autres dits savants. Ce qui tend à prouver, contrairement à une idée reçue, non dite, qu'on n'écrit pas une grammaire pour un public universel, mais toujours pour un public particulier.

Je voudrais donc présenter ma conception d'une "grammaire du sens et de l'expression" à partir d'un certain enjeu éducatif ${ }^{3}$, pour montrer comment celui-ci peut trouver dans le savoir savant des éléments de réponse. Car je ne crois pas que, dans l'enseignement scolaire, on puisse appliquer de bout en bout une théorie linguistique (ou une grammaire) ${ }^{4}$

\section{L'enjeu éducatif}

Partons donc de l'idée qu'on ne peut aborder les questions de contenu d'une discipline sans s'interroger sur les conditions sociales de son enseignement. Les conditions sociales de l'enseignement ne résident pas uniquement dans l'appareillage administratif qui préside à l'organisation de l'école et à l'exercice du métier d'enseignant. Même si l'on sait que ces conditions sont importantes, il en est d'autres qui sont davantage liées à l'activité d'enseignement-apprentissage elle-même. Si tout récemment des propositions ont été faites pour améliorer le fonctionnement du collège, la question des contenus reste entière. C'est que l'enseignement d'une discipline, dont les deux composantes - contenus et méthodes - sont indissolublement liées, dépend des "représentations" de valeur, d'utilité et de faisabilité qu'en ont ses acteurs. Evidemment ces représentations 
5 Faute de cette condition de socialité on observe un décalage entre la formation universitaire, la formation des maîtres et la réalité de la classe, chacune de ces parties «imaginant» ce que devrait faire l'autre. ne sont pas les mêmes selon qu'il s'agit des enseignants, des élèves, des parents ou de l'administration, mais ce sont elles qui pèsent de tout leur poids sur l'enseignement dans son intentionnalité, dans sa mise en œuvre et dans son résultat, d'un poids qui explique les échecs ou assure les réussites.

Parmi cet ensemble de conditions, j'en vois trois qui me paraissent essentielles pour l'enseignement de la grammaire: - une condition d'applicabilité. Accepter que l'école, y compris dans les secteurs les plus spécialisés, n'est jamais le seul lieu de construction du savoir ni du savoir faire. S'agissant de l'aptitude à parler, à communiquer et à écrire, par exemple, il n'a jamais été prouvé que cela se faisait grâce aux exercices scolaires. Hors l'apprentissage de base de la lecture et de l'écriture, ce ne sont pas nécessairement les leçons de grammaire et de vocabulaire, les rédactions et les dissertations, les explications et autres commentaires de textes qui donnent ces aptitudes. D'ailleurs, pourquoi voit-on certains élèves faire mieux que d'autres, alors qu'on leur fournit les mêmes instruments? pourquoi les élèves les plus performants sont ceux qui vivent dans un environnement où sont socialement valorisés la lecture et l'écriture? pourquoi le changement de méthode pédagogique ne bouleverse pas cette hiérarchie? pourquoi existe-t-il des poètes, écrivains, conteurs, parfois autodidactes, qui témoignent qu'ils ont appris à écrire par la fréquentation des écrits les plus divers alors qu'ils étaient parfois (souvent?) des élèves moyens ou médiocres? ${ }^{5}$ Autrement dit, on n'est jamais assuré que la bonne connaissance du fonctionnement d'un système améliore le savoir parler et écrire. De même qu'il n'est point besoin d'être mécanicien pour conduire une voiture, il n'est pas nécessaire de savoir analyser une langue pour bien s'exprimer.

- une condition de filiation. Tenir compte de l'héritage social en matière de savoir. On ne peut passer d'un système d'enseignement à un autre, d'un type de savoir à un autre, d'un type de savoir-faire à un autre, sans tenir compte de ce qui a été déjà acquis par l'élève, non seulement par lui-même dans son passé, mais aussi par les adultes qui l'entourent et qui lui renvoient une image de ces savoirs et savoir-faire. Il est nécessaire que ces images ne soient pas en contradiction les unes avec les autres, car ces savoirs ne pourraient plus circuler entre les générations, la solidarité si nécessaire à la cohésion sociale ne pourrait plus s'exercer, et la société, ne pouvant plus 
6 Evidemment, on ne retiendra pas l'argument qui consiste à dire que "de mon temps on a bien enseigné et appris selon des méthodes traditionnelles, pourquoi celles-ci ne s'appliqueraientelles pas aux jeunes générations ?". Réponse : "parce que ni la société ni les conditions d'enseignement ne sont les mêmes".

7 Est visé ici le fait que certains Instituts de Formation des Maîtres (IUFM), en France, minimisent l'enseignement des disciplines au profit d'une interdisciplinarité plus perturbante que formatrice.

8 Il y a d'ailleurs eu, au cours de l'histoire, diverses descriptions grammaticales avec des objectifs différents, les uns plus tournés vers des catégories logiques comme la grammaire de Port-Royal, d'autres plus tournés vers des catégories notionnelles comme la grammaire de F. Brunot. se retrouver dans un patrimoine commun, perdrait beaucoup de son identité. Il faut donc respecter la tradition non pour des raisons morales, ni même éthiques, mais pour des raisons biologiques, affectives et intellectives. Les processus éducatifs évoluent lentement dans un jeu d'intégration progressif de la nouveauté sans perte immédiate de la tradition ; il faut éviter que l'innovation, si nécessaire, ne corresponde davantage à une fuite en avant qu'à un progrès ${ }^{6}$.

- une condition de disciplinarité. Ne pas perdre de vue que toute construction d'un savoir passe par l'acquisition d'un "savoir dire", et que tout savoir dire est dépendant d'une discipline qui s'est forgée un corps de concepts nécessaire à la compréhension des phénomènes qui sont étudiés par celle-ci. Il n'est guère d'enseignement possible, dans quelque domaine que ce soit - du plus manuel et artisanal au plus spéculatif-, qui ne soit organisé en discipline. Et même si l'on veut faire de l'inter-, pluri- ou trans-disciplinaire, ce n'est que dans la mesure où l'on a au préalable des connaissances dans différentes disciplines qu'on l'on pourra les mettre en relation. L'interdisciplinarité ne peut être qu'un point d'arrivée et non un point de départ. Il faut éviter de faire croire aux enseignants en formation que la solution aux problèmes de l'enseignement de la langue se trouve hors de leur discipline de référence ${ }^{7}$.

\section{Propositions}

Proposer une nouvelle façon d'enseigner la grammaire suppose que l'on ait constaté des manques, voire des contradictions, dans les autres façons de faire. La tradition, en tant que telle, n'est pas ici en cause ${ }^{8}$. Ce que je voudrais opposer ici ce sont deux conceptions opposées: l'une qui est tournée vers la description des formes de la langue, et qu'on appellera "grammaire morphologique", l'autre qui est tournée vers le sens et qu'on appellera "grammaire sémantique". Il faut reconnaître que ce qui a prévalu - et prévaut encore- dans la tradition scolaire a été - et est- une grammaire centrée sur la découverte et la description des formes: la formation des mots, une catégorisation en parties du discours (morphologie) autour de catégories proprement grammaticales (substantif, adjectif, verbe, adverbe), les règles de combinaison de ces formes et de construction des phrases (syntaxe) en opérant une hiérarchie 
entre ce qui est le plus important (propositions principales) et ce qui est jugé secondaire (propositions subordonnées). Dans une telle conception de l'enseignement de la grammaire, la langue est présentée comme un ensemble de formes qu'il s'agit de maîtriser. Cela est parfaitement justifié pour l'enseignementapprentissage de l'orthographe, mais cette conception, qui s'impose pour des enfants en voie de scolarisation, perdure malheureusement au-delà de l'enseignement primaire. Ainsi voit-on dans les manuels de grammaire -et ce malgré des habillages modernistes au nom de la communication et de l'expression- des leçons sur: la nature et la fonction des mots ; les propositions relatives qu'il faut apprendre à distinguer des conjonctives; la conjugaison, les temps des verbes et les accords du participe ; la différence entre Cod, Coi, Cos ; l'attribut du sujet qu'il ne faut pas confondre avec l'attribut du Cod, etc. De plus, dans le traitement de ces formes et de leurs règles de combinaison, ne sont pas traités les problèmes de la différence entre: "Jean fume la pipe", “Jean fume sa pipe" et "La cheminée fume", ou bien entre "Jean franchit le pont" et "Le pont franchit la rivière", phrases dans lesquelles le statut du sujet et de l'objet n'est pas identique. De même, sont confondus le mode "conditionnel" comme expression de la condition, alors qu'il exprime bien d'autres choses que la condition, et la notion de condition qui peut être exprimé par autre chose que le conditionnel. Enfin, les "déterminants" sont présentés comme ce qui "précède le nom", puis décrits du point de vue de leurs formes, sans que soient mis en lumière les enjeux d'expression possibles qui résultent de l'emploi de telle forme d'article, de démonstratif, de possessif ou d'indéfini. On pourrait relever de nombreux exemples de la sorte, et montrer que des réponses existent pour résoudre ces problèmes, si tant est qu'on les aborde en s'interrogeant sur la finalité sémantique de ces catégories.

Voyons donc ce que serait une grammaire du sens qui, disons-le tout de suite, ne nie pas l'existence des formes ni la nécessité de respecter les règles de construction, mais cherche à mettre en relation ces formes avec ce qu'elles signifient, en fonction de ce au service de quoi elles sont employées. S'il y a rupture dans la façon d'aborder la grammaire, ce n'est pas visà-vis des formes mais dans la conception même du traitement des formes. La rupture est conceptuelle.

Trois options caractérisent une telle grammaire. 


\section{Une grammaire de l'énonciation}

La langue doit être décrite du point de vue de catégories qui correspondent à des intentions de communication (le sens), en mettant en regard de chacune d'elles les moyens (les formes) qui permettent de l'exprimer.

Ainsi est-on amené à décrire la langue à partir des opérations conceptuelles que fait le sujet parlant quand il communique, et non à partir des catégories morphologiques. Les déterminants (articles, possessifs, démonstratifs) sont des catégories de forme et non d'intention. C'est l'opération: "identifier les êtres du monde dont on parle" qui correspond à une intention et donc à une catégorie de sens. L'article, le possessif et le démonstratif ne sont que des moyens formels parmi d'autres d'exprimer cette intention, chacun ayant sa spécificité. Le pluriel n'est pas davantage une catégorie de sens. C'est $l^{\prime \prime \prime}$ intention de quantifier les êtres du monde dont on parle" qui l'est, et c'est pour exprimer cette intention que nous disposons de quantificateurs tels les indéfinis, les adverbes de quantité, les adjectif numéraux, et diverses expressions figées. Le pluriel n'est qu'affaire d'accord morphologique. D'ailleurs, comment expliquer, si l'on s'en tient aux seules catégories de forme, que le singulier, en français, puisse servir à exprimer une grande quantité ("Il y a de la voiture à Paris") ${ }^{9}$ ? Au lieu de présenter la description des catégories en entrant par les parties du discours, pourrait-on entrer par l'intention de communication à laquelle elles correspondent ("actualisation", "dépendance", "désignation", etc.), et construire de la sorte une grammaire du sujet parlant dont l'objectif est d'exprimer des intentions à l'aide de certaines formes.

Cela conduit à opérer un regroupement des catégories de formes autour de différentes intentions de communication qui font éclater la structure de la grammaire morphologique. Par exemple, les adverbes de quantité, les adjectifs indéfinis, les

9 Pourtant, il est aisé d'expliquer pourquoi, $\mathrm{du}$ point de vue du sens, une forme partitive au singulier, appliquée à des objets dénombrables, exprime une «quantité massive» et donc produit un effet de grande quantité. déterminants au pluriel (ainsi que le partitif) et des expressions diverses seront regroupés sous la catégorie "quantification" ; les adjectifs qualificatifs, certains compléments de nom, certains adverbes, ainsi que les propositions relatives, participes et gérondives seront regroupés sous la catégorie "qualification" (voir cidessous la structuration). 
Une grammaire des effets de discours

On ne se contentera pas de la définition des catégories, et on montrera que les formes qui servent à les exprimer produisent des effets de sens particuliers selon qu'elles sont employées dans tel ou tel contexte, dans telle ou telle situation.

Par exemple, l'article le sert à "identifier", mais ce faisant il permet d'exprimer des effets de discours particuliers: de familiarité ("On se retrouve au bistrot du coin"), ou d'antonomase, "le X par excellence", comme dans un menu ("Le confit de canard"). L'article un, en revanche, produira un effet de singularité ou d'unicité comme dans un inventaire ou dans "j'ai pêché une carpe de $3 \mathrm{~kg}$ ". Quant à l'article zéro, il produira un effet d'étiquetage comme dans les titres de journaux, les annonces ou dans "Confit de canard". Dès lors, on voit que l'article, tout en jouant son rôle linguistique d"'identification" des êtres, peut aussi tantôt placer l'interlocuteur dans une certaine relation polémique vis-à-vis du sujet parlant, puisque le met l'interlocuteur en lieu et place de quelqu'un qui sait, alors que un le met en lieu et place de quelqu'un qui ne sait pas, tantôt produire un effet de prestige ou au contraire de banalisation. Il en est de même pour le pronom on. Cet indéfini, avec sa valeur d'impersonnalisation peut produire des effets de discours de distanciation ("Je vois qu'on ne maime pas beaucoup"), d'affection ("Alors, on travaille dur, mon petit?"), de déresponsabilisation ("Salut, ça va? - Bof, on fait aller"), voire de mépris, à moins que ce ne soit d'ironie ("On ne pense pas beaucoup, ici, à ce que je vois !"). Mais on pourrait aussi montrer en quoi les constructions de phrases actives, passives et nominalisées correspondent à des enjeux d'expression particuliers, ce que l'on voit par exemple dans les titres de journaux ("Une voiture a renversé un piéton" / "Un piéton a été renversé par une voiture" / "Un piéton, victime d'une voiture").

Ainsi, pourra-t-on faire comprendre que les catégories linguistiques sont au service du discours et que, du même coup, parler correspond à un enjeu social qui consiste d'une part à s'ajuster à des normes d'usage, d'autre part à jouer avec ces normes. Parler n'est plus affaire d'esthétique mais d'ajustement, d'adéquation et de stratégie. 


\section{Une grammaire pour réfléchir}

Toute grammaire est explicative. Mais il y a plusieurs façons d'expliquer. Il y a des explications historiques qui consistent à expliquer l'état d'un phénomène par son origine et donc un état de la langue par son étymologie ; des explications fonctionnalistes qui consistent à expliquer comment se forment les mots (morphologie et dérivation) et selon quelles règles ils s'agencent; des explications sémantiques qui consistent à expliquer les différences de sens entre différentes façons d'exprimer une même intention. Ce dernier type d'explication s'inscrit dans une problématique de l'intentionnalité et de la pertinence, dans la ligne de la philosophie du langage. Dans ce dernier cas, expliquer revient à tenter de faire comprendre à la fois la vision du monde que propose une catégorie et les multiples effets de sens qu'elle est susceptible d'engendrer.

Nous avons vu que l'intention de "quantifier" pouvait être exprimée de diverses façons, et donc elle peut faire l'objet d'autant d'explications qui correspondent à ces différents sens. Mais on pourrait prendre aussi le cas des possessifs, et faire réfléchir sur les différents sens du lien de "possession". a-t-il possession (ou possession identique ) dans: "Sa tête va exploser", "Voici sa voiture", "Je te présente sa femme", "Sa passion est extrême", "Son style ne me plaît pas"? On montrera alors ce qu'il y a de commun à ces énoncés l'opération de "mise en interdépendance de deux éléments), et la différence des liens qui relient ces éléments: le premier est de "nature, le second d'"appropriation", le troisième de "parenté", etc. On pourrait également faire réfléchir sur l'interrogation qui n'est pas seulement affaire de forme (différences entre la forme directe, l'inversion ou est-ce que), mais de vision du monde quant à la façon dont les êtres sociaux entrent en relation les uns avec les autres, et établissent des rapports de force (d'imposition ou de sollicitation vis-à-vis de l'autre) en exprimant un ordre déguisé, de l'étonnement ou de l'indignation.

Il s'agit donc d'expliquer pour faire réfléchir, ce qui est fondamentalement le rôle de l'école, alors que l'apprentissage de formes et de règles déliées de leur finalité n'apporte aucune possibilité de réflexion. Evidemment, cela suppose que l'on opère quelques changements terminologiques, comme celui d'introduire la notion d'"actant" si l'on veut 
traiter la question du sujet grammatical et des compléments d'objet directs et indirects. En fait, pour régler ces problèmes, les innovations terminologiques sont peu nombreuses, le tout étant d'introduire celles qui sont indispensables à la production d'une explication utile.

\section{Une structuration de la grammaire différente}

Reste la question de savoir si l'on peut procéder à une catégorisation d'ensemble d'une telle grammaire.

Il a toujours existé dans la linguistique des courants qui cherchent à déterminer des catégories sémantiques qui correspondraient à des catégories de pensée ou d'intention. Filiation sémantique qui a été en partie occultée par la grammaire générative puis par certains modèles cognitivistes. Ce sont certaines théories nées dans la mouvance du structuralisme avec des linguistes qui ont travaillé plus particulièrement sur les "temps des verbes" (G. Guillaume, H. Weinrich, J. Fourquet), les "éléments de relation" (B. Pottier), les "modalités" (J. Lyons, B. Pottier), les "quantificateurs" (O. Ducrot, R. Martin) ; les théories "casuelles" (Ch.J. Fillmore, J.M. Anderson) qui ont repris la question des constructions de phrases autour des notions d'actants et de types de relation entre les actants, et que l'on retrouve dans certaines théories fonctionnalistes américaines ; la théorie de l'"énonciation" qui s'est attachée à décrire plus particulièrement le fonctionnement de la "deixis" (E. Benveniste, A. Culioli) ; la théorie des "actes de parole" initiée par J.L. Austin et J. Searle qui a trouvé de nombreux prolongements dans les études des cognitivistes. Les catégories qui sont issues de ces approches ne cessent d'être discutées autour de la question de savoir si elles peuvent être déclarées universelles, si elles correspondent à des catégories de pensée (indépendantes des langues) ou à des catégories strictement linguistiques.

Je n'entrerai pas, ici, dans ce débat, mais je voudrais montrer comment on peut justifier une structuration de la langue en catégories conceptuelles dont on peut faire l'hypothèse qu'elles correspondent à des intentions de communication. Je partirai pour ce faire d'un exemple. Imaginez que quelqu'un vous dise, comme ça, de but en blanc: "Cheminée". Vous serez en droit de lui rétorquer: "Et alors?". Qu'il vous en dise un peu plus: “Une cheminé", et vous 
continuerez à lui demander: "Et alors?". S'il vous en dit encore un peu plus avec un timide: "Une grande cheminée", vous serez encore insatisfait et lui renverrez un autre: "Et alors?" peutêtre un peu agacé. Qu'il se lance alors dans un: "Une grande cheminée est tombée", et vous commencerez à entrevoir qu'il veut vous raconter un événement qui s'est déjà produit, mais vous ne voyez toujours pas où il veut en venir. Il précisera peut-être: "Une grande cheminée est tombée hier place de la Bastille", ce qui provoquera peut-être votre réaction: "Ah bon, et alors?". Et il terminera par un: "Trois blessés, enfin, je crois". Trois enseignements peuvent être tirés de cette petite saynète.

Le premier est que l'unité communicative n'est pas forcément la phrase, comme on le dit parfois, mais un ensemble de d'énoncés dont la combinaison permet à l'interlocuteur de voir de quoi il retourne. Et de fait, on est toujours fondé à répliquer "Et alors?" à quelqu'un qui vous parle tant que l'on ne comprend pas de quoi il s'agit, c'est-à-dire tant qu'on n'arrive pas à imaginer ce qu'est son intention de communication. C'est ce que j'appelle, dans mon jargon d'analyste du discours une "problématisation". Le rapport thème/prédicat correspond peut-être à une unité de phrase (ou d'énoncé, selon le point de vue que l'on prend), mais il ne garantit pas que l'on ait affaire à une intention communicative. Pour être un peu plus technique, on peut dire que pour parler et se faire comprendre il est certes nécessaire de "thématiser", mais non suffisant, car il faut encore "problématiser" son propos: c'est la problématisation du discours qui construit, et fait comprendre, l'enjeu qui s'attache à l'intention de communication.

Le deuxième enseignement a trait à la possibilité de découvrir quelles sont les catégories conceptuelles auxquelles le sujet parlant fait appel pour construire son discours. D'une part, il faut bien nommer les "êtres" du monde. Que ceux-ci soit des êtres animés ou pas, humains ou pas, matériels et concrets ou abstraits et imaginés, il faut leur donner un corps qui les configure linguistiquement, c'est-à-dire les "nommer". On dispose, pour cela, du moins dans nos langues romanes, de noms propres et de noms communs. C'est le cas de "cheminée". D'autre part, ces êtres du monde sont entre eux différents et en même temps ressemblants pour diverses raisons. Il faut les déterminer quant à leur actualisation, leur quantité, leur appartenance, etc.) afin de les "identifier" ("Une cheminée"), 
mais il faut aussi préciser quelles sont leurs propriétés, autrement dit les "qualifier" ("Une grande cheminée"). On a vu cependant, que cela n'était pas ici suffisant pour saisir l'enjeu de l'acte de communication. Il fallait encore que l'on dise dans quel "cadre événementiel" s'inscrivent ces êtres ainsi identifiés avec leurs propriétés ("Une grande cheminée est tombée"). Ensuite, peuvent être précisés le lieu et le temps dans lequel se "situe" l'événement ("Une grande cheminée est tombée hier place de la Bastille"). Et pourtant, cela n'est pas encore suffisant pour que l'on soit complètement satisfait, car il faut encore que l'on puisse percevoir dans quelle "chaîne de causalité" (cause/conséquence) s'inscrit cet événement "Trois blessés"). Enfin, même si l'on peut considérer que le minimum du contrat communicatif a été rempli, on sait bien que tout acte de langage dépend, pour sa valeur de vérité, du "point de vue" de celui qui l'énonce. Ce point de vue peut rester implicite, ne pas être dit, mais on ne peut y échapper, et il peut même être explicité comme c'est le cas dans notre exemple par une certaine façon de modaliser son propos ("enfin, je crois"). Ainsi se justifie une catégorisation qui constitue la base de cette grammaire de l'énonciation dont j'ai parlé, puisqu'elle est construite autour d'opérations langagières qui correspondent à autant d'intentions de communication. Et c'est sur la base de cette catégorisation que sont regroupées les catégories de formes de la grammaire morphologique, selon le modèle suivant: nommer les êtres du monde ("noms propres" et "noms communs"), les identifier ("déterminants" et "quantificateurs"), les qualifier (différents processus d'"adjectivation"), décrire les événements dans lesquels ils s'inscrivent (les verbe d'"action" ou de "fait" ${ }^{10}$ ), les situer dans l'espace et dans le temps ("prépositions", "adverbes", "temps et aspects verbaux"), donner les motifs et/ou les conséquences de ces actions ("conjonctions" et "propositions" de "cause", de "but", de "conséquence"), enfin préciser qui parle et quel est son point de vue (la "pronoms", les "modes", les adverbes et verbes de "modalité").

Le troisième enseignement s'appuie sur les deux précédents. Tout acte de langage participe d'un acte de

${ }_{10}$ Pour cette distinction, voir ma Grammaire du sens et de l'expression, Hachette, Paris, 1992, p.31. communication, et tout acte de communication est destiné à faire sens entre les interlocuteurs qui en sont partie prenante. Et pour qu'un acte de langage fasse sens, il faut que le discours 
produit renferme une (ou plusieurs) problématisations. Or, la problématisation est le résultat d'une certaine combinaison entre les différentes catégories de base que nous avons décrites, et que nous résumerons en disant qu'il faut minimalement: décrire, raconter, argumenter et modaliser ${ }^{11}$.

Intenção

Operações de Língua

Categorias de Forma

Modo de Organ. do Discurso

\begin{tabular}{|c|c|c|c|}
\hline NOMEAR & A Denominação & Os Nomes (N. próprio/N. Comum) & \multirow[b]{4}{*}{ O DESCRITIVO } \\
\hline \multirow{3}{*}{$\begin{array}{l}\text { DETERMINAR } \\
\text { (Modo de identificação) }\end{array}$} & A Pessoa & Os Pronomes pessoais & \\
\hline & $\begin{array}{l}\text { A Atualização } \\
\text { A Designação } \\
\text { A Dependência }\end{array}$ & $\mid \begin{array}{l}\text { Os Artigos } \\
\text { Os Demonstrativos } \\
\text { Os Possessivos }\end{array}$ & \\
\hline & $\begin{array}{l}\text { A Quantificação } \\
\text { A Identificação. indeterminada } \\
\text { A Presentação }\end{array}$ & $\begin{array}{l}\text { Os Quantificadores } \\
\text { Os Indefinidos } \\
\text { Os Apresentadores }\end{array}$ & \\
\hline $\begin{array}{c}\text { QUALIFICAR } \\
\text { (Maneira de ser/fazer) }\end{array}$ & $\begin{array}{l}\text { Definir } \\
\text { Caracterizar } \\
\text { (de maneira Objetiva/ Subjetiva) }\end{array}$ & $\begin{array}{l}\text { Os Nomes } \\
\text { Os Adjetivos } \\
\text { Os Relativos } \\
\text { Os Particípios }\end{array}$ & \multirow{3}{*}{ O NARRATIVO } \\
\hline $\begin{array}{l}\text { DESCREVER } \\
\text { A AÇÃ̃O }\end{array}$ & $\begin{array}{l}\text { Os Actantes (Agente, Paciente, etc.) } \\
\text { As Relações actanciais } \\
\text { As Configurações (Ativo/Passivo/Nom. }\end{array}$ & Os tipos de construções & \\
\hline $\begin{array}{c}\text { SITUAR } \\
\text { (Estruturação do } \\
\text { Espaço-Tempo) }\end{array}$ & $\begin{array}{l}\text { A Localização no espaço } \\
\text { A Situação no tempo }\end{array}$ & $\begin{array}{l}\text { As Preposições } \\
\text { Os Advérbios e Indicadores de tempo/esp. } \\
\text { Os Tempos e Aspectos dos verbos }\end{array}$ & \\
\hline $\begin{array}{c}\text { EXPLICAR } \\
\text { (As relações lógicas }\end{array}$ & $\begin{array}{l}\text { A Causalidade (causa/consequiência) } \\
\text { A Restrição e a Oposiçãa } \\
\text { A Condição/a Hipótese }\end{array}$ & $\begin{array}{l}\text { As Conjunções } \\
\text { As Proposições/orações }\end{array}$ & O ARGUMENTATIVO \\
\hline $\begin{array}{c}\text { DIZER O } \\
\text { PUNTO DE VISTA } \\
\left(\begin{array}{c}\text { O posicionamento } \\
\text { do Sujeito })\end{array}\right. \\
\end{array}$ & $\begin{array}{l}\text { A Afirmação e a Negação } \\
\text { A Modalização }\end{array}$ & $\begin{array}{l}\text { As Formas positivas e negativas } \\
\text { Os Modos do verbo } \\
\text { As marcas de modalidade (verbos e adv.) }\end{array}$ & O ENUNCIATIVO \\
\hline
\end{tabular}

${ }_{11}$ C'est pourquoi la $3^{\text {ème }}$ partie de la Grammaire $d u$ sens et de l'expression, op.cit., est consacrée aux différents "modes d'organisation du discours : énonciatif, descriptif, narratif et argumentatif".

\section{Conclusion}

Une grammaire sémantique est un instrument parmi d'autres permettant de répondre aux problèmes d'enseignement du sens, non comme un deuxième temps dans le processus d'apprentissage, mais comme un accompagnement de la découverte et du maniement des formes.

L'utilisation d'une telle grammaire dans l'enseignement permet en outre de mettre l'élève en lieu et place d'un sujet parlant qui ayant une intention de sens se demanderait de quels moyens formels il dispose pour l'exprimer, et quelles différences de sens s'attachent à chacune des formes dont il 
${ }^{12} \mathrm{~L}$ a série d e s grammaires de chez Hatier $\left(6^{\circ}, 5^{\circ}, 4^{\circ}\right.$ et $\left.3^{\circ}\right)$ est, à ma connaisance, la seule qui propose de véritables entrées dans les activités langagières, à partir de ces modes d'organisation du discours.

${ }^{13} \mathrm{Y}$ compris pour l'orthographe dont l'enjeu social est la reconnaissance de qualification, de savoir faire, voire de prestige. dispose. Ainsi, il pourrait à la fois faire œuvre de conformité aux conditions d'emploi dans lesquelles il se trouve, et œuvre de singularité dans sa façon de s'exprimer ce qui devrait lui faire prendre conscience de ce qu'il est en tant qu'être social et singulier.

Du point de vue pédagogique, cela devrait permettre de donner un enjeu social à l'apprentissage de la grammaire: comment enseigner la langue, non plus pour elle-même (savoir repérer et analyser des formes), mais pour faire comprendre que les différentes catégories de la langue sont au service d'un mécanisme d'expression qui permet de décrire, raconter et argumenter $^{12}$. De plus, cela devrait permettre de mieux différencier cet enseignement en fonction des différents stades d'apprentissage en l'accompagnant d'une pédagogie appropriée pour chacun de ces stades: l'enseignement primaire, étape de découverte de la manipulation des formes en relation avec leurs sens, première entrée dans l'apprentissage des normes sociales du langage ; une première étape du secondaire, où l'élève découvre les normes discursives, c'est-à-dire des différents "genres de discours", et les marges de manœuvre qui s'offrent à lui ; une deuxièmes étape du secondaire, qui complète les précédentes, et où l'on apprend à diversifier les possibilités d'expression, à passer d'un genre à un autre, à faire montre de création dans différentes situations de communication, à développer son pouvoir argumentatif. Une fois de plus l'enseignement et l'apprentissage requièrent des actes pluriels: il est un temps pour le repérage et la stabilisation des formes, un temps pour la découverte des règles de syntaxe, un temps pour la découverte du sens, mais sans perdre de vue qu'à chacun de ces moments correspond un enjeu de communication sociale ${ }^{13}$.

Encore faut-il que l'enseignant ait connaissance de ces différents types d'organisation de la langue, et pour ce qui concerne le sens, ait connaissance des mécanismes sémantiques de la mise en discours. Cela est à la fois affaire de formation initiale et de formation continue pour éviter qu'il y ait une trop grande distorsion entre la formation des maîtres et les pratiques de la classe. Mais est-il possible de se faire entendre au milieu d'une polémique qui ne fait que reproduire une querelle des anciens et des modernes dont le résultat est quelque peu stérile? 


\section{Abstract}

\section{For the teaching of a grammar based on meaning}

This article presents the conception of a "grammar based on meaning and expression" ("enunciative", "discursive", "reflexive") as a purpose for application of linguistic theory in language teaching (mother or foreign language) at schools, focusing on the development of student's linguistic proficiency. This paper also aims at opposing "semantic grammar", which emphasizes meaning, to "morphological grammar", which focus on the "description of linguistic forms", in order to show the need for reflection at school, on semantic purpose of linguistic resources and relationship between form and that which they mean according to their use. Finally, the author defends the analysis of linguistic categories as a mechanism of expression that allows to describe, narrate and argument based on the communicative situation.

Keywords: Semantic grammar. Language teaching. Linguistic-discursive resources. 